\title{
An examination of the profile and journey of patients with mental illness in the Emergency Department
}

\section{Abstract}

Objective: To examine the profile and journey of patients with mental illness in the emergency departments (ED) of a Local Health District in Australia. There is limited evidence around the patient profile and journey of people in who present to EDs with mental illness, and the aim of this study was to inform the development of a psychiatric emergency service model.

Methods: The study design was a retrospective descriptive analysis of routinely collected data of two hospital EDs. Retrospective health care data of patients who presented to the EDs between July 2016 and June 2017 was analysed using descriptive and inferential statistics.

Results: Mental health presentations ( $\mathrm{N}=4506)$ consisted of $3.44 \%$ of all presentations to the Emergency Department, mostly for anxiety and stress, comorbid alcohol and drug issues, and suicidality. Females were more likely to present, and significantly more likely to present multiple times (specifically for age groups 12-17 and over 65). Presenting issues were associated with age and gender for most diagnostic groups, but not with number of presentations, except for personality disorder. $24.83 \%$ were admitted to a mental health inpatient unit, $54.34 \%$ were referred to a mental health community team, $4.63 \%$ medically, and the remaining referred to alcohol and drug services, GPs, or NGOs. Length of stay was associated with gender (female), age $(>65)$ and diagnosis (drug related and eating disorder).

Conclusion: There is limited evidence around the patient profile and journey of people who present to EDs for mental health reasons, and this study contributes to this literature.

Keywords: mental health, emergency department, gender variation, age variation, length of stay, patient characteristics, referral destination. 


\section{Introduction}

Hospital emergency departments (EDs) play a role in treating people with mental illness (1, 2). Despite efforts to care for people with mental illness in the community, away from hospitals, people with mental illness continue to present to EDs with both emergency and non-emergency mental health problems (3-5). EDs are used by people seeking mental health $(\mathrm{MH})$ services for the first time as well as those already receiving $\mathrm{MH}$ care in the community who require an urgent or immediate response $(2,3)$. The way in which mental health care is provided in EDs can vary significantly, and different models of care exist (for a review see Coates (1)).

The number of patients who present to public hospital EDs is growing, nationally and internationally (6-9). An Australian study by Tankel, Di Palma (8) indicates that the growth rate for $\mathrm{MH}$ presentations to EDs is double the growth rate for overall ED presentations. While estimates vary considerably, studies indicate that between three and a half and nine percent of all presentations to public hospital EDs are from people seeking $\mathrm{MH}$ care $(6,8$, $10,11)$. The effective management of people who present to EDs for $\mathrm{MH}$ reasons is an issue of considerable interest, in Australia and globally $(3,12)$. Mental health presentations tend to cause issues around patient flow in ED; they generally take much longer to assess and ED staff commonly report not feeling equipped to treat this patient group (12-14). While $\mathrm{MH}$ services are expected to implement models of care that streamline transition through the $\operatorname{ED}(11,15)$, there is a lack of clarity around what constitutes best-practice in this space $(1,3$, 16).

To inform the development of a psychiatric emergency service model, patient and service data of an ED MH service was analysed of a Local Health District (LHD) in a peri-urban area of New South Wales, Australia, over a one year period. The aim of this study was to gain insight into the characteristics and patient journey of people who present to ED with mental health presentations, and examine similarities and differences based on gender, age, diagnosis, the frequency of presentations and length of stay (LOS).

There is limited evidence around the patient profile and journey of people who present to ED with mental health reasons, and the limited literature that is available tends to present inconsistent findings. In terms of patient characteristics, while some studies indicate that more females present to $\operatorname{ED}(6,11)$, other studies have found that males are more likely to present (9). While some studies indicate that a high proportion of mental health presentations are for comorbid drug and alcohol problems (9), other studies do not highlight this population as prevalent (11). While there is more agreement in the literature in terms of age, indicating that young people are most likely to present to $\operatorname{ED}(6,11,17)$, the age of people who presented to EDs for mental health reasons was an issue of considerable interest locally. There was debate amongst mental health and ED managers around the extent to which young people in our local area required emergency psychiatric care, and what the resource requirement for this is. Furthermore, with the exception of Lui, Ali (18) to the best of our knowledge there are no studies specific to ED presentation for children under the age of 12, and the emergency mental health needs of this group was identified as a key consideration locally. With the exception of a study by Penfold, Groll (19) specific to 
personality disorder, we could not find any studies that investigated frequency of presentations in relation to gender, age, and diagnosis. In relation to the LOS for mental health presentations in the ED, the average LOS reported by studies ranges significant; a study by Knott, Pleban (12) reported the average LOS as 4.5 hours (12), whereas Weiss, Chang (20) reported the average LOS as 8.2.

The aim of the current study was to inform a psychiatric emergency model of care, including targeted interventions and strategies that addressed the needs of our population. For example, we wanted to develop hospital avoidance strategies, and wondered which populations to target. While there tends to be a focus on those patients who present most frequently, "frequent flyers" (21), we wondered whether these patients are more likely to lead to delays in ED, and have a longer LOS, than patients who present less frequently. Furthermore, we were interested in the association between frequency of presentation and gender, age, and diagnosis to inform the development of targeted interventions. We also wanted to gain insight into the patient journey, to inform strategies to provide care in the community, or the least restrictive environment appropriate.

\section{Method}

The study design was a retrospective descriptive analysis of routinely collected data over a one year period, July 2016 and June 2017.

\section{Service setting}

The LHD in which this study was completed encompasses two hospitals with two EDs, providing care to a population of 325,082 . Patients can access emergency care by selfpresenting to the ED or being transported to ED via ambulance or police. In Australia, police and ambulance personnel can transport people to ED for a MH assessment against their will if they are deemed at risk and not able to make an informed decision about their care (under the New South Wales Mental Health Act (2007)).

A team of specialist $\mathrm{MH}$ nurses, supported by a psychiatry registrar and consultant, provides in-reach into the ED 24/7 to conduct $\mathrm{MH}$ assessments (for presentations identified as $\mathrm{MH}$ at ED triage), facilitate admissions to $\mathrm{MH}$ inpatient units (MHIPUs) and make referrals to community teams for patient follow up.

\section{Data Collection}

This is a retrospective study, using existing health data collected as part of routine care. In Australian hospitals and community settings patient information is maintained electronically in the patient's electronic medical record (eMR) (22). eMR data is commonly supplemented by datasets that are manually collected by clinicians. In the EDs under investigation, patient and service data was entered into eMR and as well as an Excel spreadsheet maintained by $\mathrm{MH}$ clinicians. The manually collected dataset duplicates some of the information captured in eMR (e.g. diagnosis), but includes additional information (e.g. involvement of police and ambulance and referral destination). 
Firstly, mental health-related ED presentations were extracted from eMR between July 2016 and June 2017 for analysis ( $N=4506)$. In eMR patient presenting problem/diagnosis is coded using SNOMED CT (Systematized Nomenclature of Medicine - Clinical Terms) (23). A SNOMED diagnosis to reflect the patient's presenting problem to ED is entered into eMR by a $\mathrm{MH}$ clinicians, usually following completion of a $\mathrm{MH}$ assessment. Presentations coded as $\mathrm{MH}$ related at discharge were extracted, as well as diagnostic codes used for drug or alcohol overdose. Drug and alcohol (D\&A) presentations often occur within the context of $\mathrm{MH}$ issues, and the majority of such presentations are referred to the $\mathrm{MH}$ team for an assessment. Accidental overdoses that did not occur co-morbidly with mental illness were excluded (e.g. accidental overdose in young children). The final data extract covered 129 unique MH SNOMED diagnostic codes (inclusive of some duplications), which the researchers grouped into 14 overarching categories to allow for data analysis (based on clinical judgment and consultation with psychiatric staff).

Secondly, to establish the validity of the eMR dataset, the dataset was compared to the manually collected data for the same period. Despite some minor variations, both datasets are consistent, indicating good data validity. As the eMR data was more complete, the eMR data is the main data source presented in this paper. The manually collected data was used as a secondary source, to present information not collected in eMR.

Lastly, a third dataset was extracted from the Community eMR for the same period consisting of 9110 patients (with 353,755 occasions of service). This dataset was linked to the ED eMR to identify the number of patients seen by a community $\mathrm{MH}$ team within six months of their presentation to ED.

\section{Data Analysis}

The data was analysed using descriptive and inferential statistics. Descriptive statistics were used to summarise the data; continuous variables are presented by medians and interquartile ranges; categorical variables are presented as percentages and frequencies.

Inferential statistics were used to test association between categorical variables. To assess associational strength for non-clustered data Pearson's chi-square test was used, with Fisher's exact test when cell counts were small (expected cell size less than 5). Significant proportions and differences between proportions were assessed respectively by the single sample proportions test and the difference of proportions test. For multiple comparisons, p-values were adjusted using Bonferroni's correction (24).

Due to non-normality, the Mann-Whitney $U$ test or Kruskal Wallis test was used to compare medians between independent groups; the former when comparing two groups and the latter when comparing more than two groups.

When data was nested within patients, binary logistic regression with a clustered variance estimator was used to adjust for correlated data. For model diagnostics, the HosmerLemeshow (25) and the Pregibon Link (26) tests were used to assess goodness-of-fit and model specification respectively. 
The level of statistical significance was set at $5 \%$ (two-sided) $(p<0.05)$ for all tests. All statistical analyses were conducted with Stata version 15.0.

$\underline{\text { Ethics }}$

Ethical approval was not required as the project was reviewed to be exempt from ethical review by a Human Research Ethics Committee and deemed an Evaluation Activity as per criteria set by NSW Health (27). The project was reviewed and authorised by the delegated representative of the organisation in line with the Health Records and Information Privacy Act (HRIP Act) (28), Ethical Considerations in Quality Assurance and Evaluation Activities developed by the National Health and Medical Research Council (NHMRC) (29) and the National Statement on Ethical Conduct in Research (2015).

\section{Results}

During the one year reporting period, $3.44 \%$ of all ED presentations across both EDs were for $\mathrm{MH}$ related issues, consisting of 4506 presentations and 3358 unique patients, with a mean age of 37.62 (ranging from 1 to 31 presentations per patient). No significant differences were identified between both sites, so the data is reported on collectively.

As outlined in Figure 1, 78.69\% ( $n=1389)$ of patients who presented in the first six months of the study period ( $\mathrm{N}=1765)$ either did not present again or presented again at a time in excess of six months. The remaining $(21.31 \%, n=376)$ presented again within six months of their first presentation. Of these, two thirds ( $n=249 ; 66.22 \%)$ presented twice. The maximum number of presentations in a six months period was 24 .

\section{Insert Figure 1}

\section{Patient characteristics}

As outlined in Table 1, more females presented to the ED than males ( $54.97 \%$ versus 45.03\%). This gender difference is most pronounced for young people aged 12 to 17 (72.41\% versus $27.59 \%)$, and older people over the age 65 (62.15\% versus $37.85 \%)$.

\section{Insert Table 1}

Table 2 outlines the association between age and gender and re-presentation. In terms of gender, females were significantly more likely to present to ED multiple times compared to males. For those patients who presented once, the proportion of females and males was fairly similar; for those patients who presented multiple times, the proportion of females is over twenty percent higher than males. In terms of age, we did not find statistically significant age differences between those who presented once versus multiple times. However, older people over the age of 65 were twice as likely to present only once versus multiple times; while not statistically significant, this may have clinical implications. 


\section{Insert Table 2}

The most common $\mathrm{MH}$ presentation was anxiety, trauma or stress related (33.49\%), followed by drug related presentations (14.47\%) (incl. overdose and drug induced psychosis), and suicidal ideation (excl. intentional overdose) (12.81\%). Anxiety and stress related conditions were the most prevalent, except for children under the age of 12 and people aged between 36-64 years. Children under the age of 12 were most likely to present with behavioural and conduct issues and people aged between 36 and 64 were most likely to present with a drug overdose. As indicated in Table 3, there is a statistically significant association between age and diagnosis, with the exception of overdose (not specified) and other/MH disorder not specified.

In terms of gender variations, females were more likely to present with anxiety and stress related conditions, deliberate drug overdose, and eating disorders. Males were more likely to present with schizophrenia and other psychotic disorders, behavioural/conduct disorders, and drug induced psychosis. There is no significant gender difference for suicidal ideation, drug overdose (not specified), depression, intentional self-harm, personality disorder, bipolar disorder, and accidental drug overdose (see Table 3 ).

There was no significant difference in diagnosis between those who presented only once versus multiple times, with the exception of personality disorder. For those patients who presented multiple times $(\mathrm{N}=376)$, the analysis of presenting issues was based on the diagnostic code allocated at their first ED presentation. The diagnostic category allocated at first and subsequent presentations is not always the same; only $38.03 \%$ of patients who presented multiple times $(n=143$ ) were allocated the same diagnostic category at first and second presentation.

\section{Insert Table 3}

\section{Patient journey}

Patients included in this sample either self-presented to ED, or were brought to ED by police and ambulance. Data for police and ambulance presentations was available for one of the EDs. Of the $2550 \mathrm{MH}$ presentations to this site during the reporting period, $20.94 \%(n=534)$ were brought in involuntarily by either police or ambulance. Around a third $(34.2 \%, n=183)$ of these were admitted to a MHIPU, which is higher than the average admission rate for $\mathrm{MH}$ presentations to the ED.

Around a quarter $(24.83 \%, \mathrm{n}=1118)$ of all MH ED presentations were admitted to the MHIPU. Just under five percent $(4.63 \%, n=209)$ were admitted medically; $54.34 \%(n=2448)$ were referred to a public $\mathrm{MH}$ community team; $2.55 \%(n=115)$ were referred to $D \& A$ services; $6.5 \%$ (293) were referred to a general Practitioner (GP), private health professional or NGO; $4.02 \%$ ( $n=181$ ) did not require further care; and 3.13\% ( $n=141)$ did not wait in the ED for a $\mathrm{MH}$ assessment to be completed. 
To determine how many patients who presented to ED were seen by a MH community team (not simply referred), the ED eMR and community eMR data were linked. This combined dataset indicates that $60.3 \%(n=2025)$ of patients who presented to the $E D(n=3358)$ had contact with a community team $(\mathrm{N}=9910)$ in the reporting period. More specifically, of those patients who presented to ED in the first six months ( $N=1765), 29.12 \%(n=514)$ had contact with a community team within six months of presenting to ED. Of patients who presented to ED in the second six months ( $N=1593), 9.35 \%(n=145)$ had contact with a community team in the six months preceding their presentation to ED. For the remaining twenty percent $(21.8 \%)$ the time between their ED presentation and community contact was in excess of six months.

Patients who accessed a community team in the six months following an ED presentation $(\mathrm{N}=514)$ were compared to those who did not $(\mathrm{N}=1251)$ in terms of gender and age. Follow up with a community team post ED presentation was associated with age, but not gender. While we found an association between age and community follow up, the sample size was too small to detect significant differences for individual age groups (see Table 4).

\section{Insert Table 4}

As indicated in Table 5, the median LOS for patients who presented for $\mathrm{MH}$ reasons was 3.43 hours. In Australia, the LOS of patients in EDs is a national performance indicator, and there is an expectation that $81 \%$ of all patients will be discharged from ED within four hours. In this study, around sixty percent (61.6\%) of patients were discharged from ED within the four hours target. Just over ten percent (10.64\%) were in ED for over eight hours, and $1.46 \%$ $(n=66)$ were in ED for over 16 hours. Differences in LOS are associated with gender, age, diagnosis and the where the patient is transferred to (mode of separation). Patients with the longer LOS were female, over 65, presented with drug related conditions or eating disorders, and/or admitted as an inpatient. There is no significant difference in terms of LOS between patients who present only once versus those that present multiple times.

\section{Insert Table 5}

\section{Discussion}

Less than four percent (3.44\%) of all presentations to the EDs were identified as $\mathrm{MH}$ related, and seen by the ED MH team. This is slightly lower than the Australian average of $3.7 \%(6)$, and significant lower than $5.3 \%$ as found by a similar Australian study by Shafiei, Gaynor (9). The lower percentage reported by the current study is likely reflective of the aging population of the local area (as older people are less likely to present to EDs for $\mathrm{MH}$ reasons).

The highest number of presentations were from people age 36-64 (36.25\%), which reflects the population demographics of the local area (30). Young people between 12 and 23 and those between 24 and 35 were most likely to present to ED with $\mathrm{MH}$ issues. While around $15 \%$ of the local population is aged between 12 and 24 (30), 31.7\% of $\mathrm{MH}$ presentations to ED where in this age range. Around 11\%, of the local population is aged between 24 and 35 
(30), whereas $21.74 \%$ of $\mathrm{MH}$ presentations to ED were in this age range. Children under the age of 12 and older people over the age of 65 were least likely to present compared to population demographics. The finding that younger people are most likely to present to ED for $\mathrm{MH}$ reasons is consistent with the literature $(6,11,17)$, and supports a recognised need for the development of crisis services for young people $(17,31)$.

Compared to males, females were more likely to present, and significantly more likely to present multiple times (specifically those aged 12-17 and over 65). While higher prevalence of females (54.97 versus 45.03) is reflective of the population demographics of the area (51.6 females versus 48.4 males) (30), the gender difference for ED presentations is greater than population gender variance. The finding that more females presented is consistent with some studies $(6,11)$, but contradicts others (9).

The most common diagnoses were anxiety and stress related (33.47\%), followed by drug overdose (12.87\%) (deliberate, accidental and not specified) and suicidality (12.81\%). The way in which presenting issues are diagnosed and coded varies across EDs nationally and internationally (23), and as such it can be difficult to compare findings; however, by and large, the current findings are in line with the literature $(6,9,11)$. Of note is the rate of comorbid $\mathrm{MH}$ and D\&A presentations identified in this study. Such presentations are not always included in similar studies (11), as the way in which these patients are captured can vary $(6,9)$ making comparison difficult. While the current study identified a seemingly high rate of comorbid $\mathrm{MH}$ and D\&A presentations in comparison to some studies (11), it is lower than the $39 \%$ identified by Shafiei, Gaynor (9). This variation can most likely be explained by the different ways in which D\&A presentations are coded, and extracted from eMR. For the current study, the $14.49 \%$ (incl. overdose and drug induced psychosis) identified in the eMR dataset was compared to the manually collected data and found to be consistent (at $16.43 \%)$. The finding that there are high rates of comorbid $\mathrm{MH}$ and D\&A presentations to ED is consistent with previous studies, which highlight a need for enhanced D\&A services in EDs (32-36).

Presenting issues were associated with age and gender for most diagnostic groups. While anxiety and stress related conditions were the most common for most age groups, people aged 36-64 were most likely to present with a drug overdose and children under the age of 12 were more likely to present with behavioural and conduct issues. Neither of these observations are surprising; the former is in line with well recognised high prevalence of drug presentations to EDs (34), and the latter is consistent with the prevalence of conduct and behavioural issues in children. However, few studies have investigated ED presentation for children under the age of 12 , and our findings contribute to this literature (18).

Gender variations in diagnoses were largely consistent with gender differences in lifetime prevalence of mental illness reported in the literature (37-39). Consistent with this literature, females were more likely to present with anxiety and stress related conditions, and males more likely to present with psychotic illnesses and behavioural issues. Of note however, is that females were more likely to present with deliberate drug overdose, and this study did not identify a significant gender difference for suicidal ideation, depression, intentional self-harm, personality disorder, bipolar disorder, and accidental drug overdose. 
In terms of the differences between people who presented once versus multiple times within a six month period, females and those diagnosed with personality disorder were statistically more likely to present multiple times. Males were significantly more likely to present only once; this does not appear to be related to gender differences in terms of diagnosis, and may be reflective of different help seeking behaviours between males and females $(40,41)$. The only diagnosis that this study detected as associated with whether people presented once or multiple times was personality disorder. However, this finding needs to be interpreted with caution as this study is limited in the extent to which it can detect an association between diagnosis and re-presentation. Given the presence of small sample sizes for some diagnoses it is likely that a number of statistical tests were underpowered. However, a significant difference was found for personality disorder, which is consistent with evidence that indicates that compared to other $\mathrm{MH}$ conditions people with personality disorders have extensive involvement with healthcare services, including EDs $(19,42)$.

A fifth of ED MH presentations were brought in by police or ambulance as involuntary patients. While this finding is consistent with the literature, it highlights the need hospital avoidance strategies to be developed conjointly by police, ambulance and $\mathrm{MH}$ services to better manage this population in the community $(10,43-46)$. Less than ten percent of ED $\mathrm{MH}$ presentations were seen by a community team within the six month preceding their ED presentation. This means that the majority of people managed by the community teams do not present to ED, and that $\mathrm{MH}$ crisis are managed in the community.

In terms of patients' referral destination, just under thirty percent were admitted to a ward ( $24.83 \%$ to MHIPU and $4.63 \%$ medically), which is lower than national average of $35.9 \%$ (6). Just over half (54.34\%) were referred to a $\mathrm{MH}$ community team, with around thirty percent were seen by a community team following their ED presentation. To identify how follow up could be improved, more research is required into the differences between those who engage with community teams following an ED presentations and those who do not. While our findings indicate that follow up is associated with age, we could not identify in what way.

In terms of LOS, around forty percent of $\mathrm{MH}$ patients were not discharged within the four hour target and over ten percent had a LOS of over eight hours. While this is concerning, the findings is consistent with the long LOS reported by other studies $(9,12,20,47-49)$. The median LOS of 3.43 reported here is lower than the median LOS reported in other studies; e.g. of 4.5 hours reported by an Australian study (12) and 8.2 hours reported by an American study (20). Longer lengths of stay were associated with gender (female), age (>65), diagnosis (deliberate drug overdose and eating disorder), and the need for an admission. The longer LOS for people over 65 and those with eating disorder and drug overdose is not surprising as these populations requires more medical involvement (comorbid physical and MH issues), which is associated with an increased LOS (20). The longer LOS for females may be indicative of the increased prevalence of this population to present with deliberate drug overdose and eating disorders. The longer LOS for patients who require an admission is also consistent with the literature and may be caused by access block, as they wait in ED for hospital bed to become available $(9,11)$. 
This study has a number of limitations, in particular the retrospective use of eMR data. As the data was collected within a clinical setting and not for research purposes the findings need to be interpreted with caution. The accuracy of the diagnostic coding is dependent on the treating clinicians' training and knowledge of the coding system. The data was based on SNOMED diagnostic codes allocated by MH clinicians to reflect the patient's presenting problem to ED, not necessarily their psychiatric diagnosis (if they had one). This has implications when interpreting the data; for example, the finding that only $38.03 \%$ of patients who presented multiple times were allocated the same diagnostic category at first and second presentation is reflective of the patient's different reasons for presenting to ED and the coding process used, and does not necessarily indicate changes to the patient's psychiatric diagnosis. This study is also limited in the extent to which it can detect diagnosis related associations due to small sample sizes for some diagnostic groups.

\section{Implications for practice}

This study describes and compares the characteristics and journey of $\mathrm{MH}$ presentations to two hospital EDs, and highlights variations across gender, age, diagnosis, the frequency of presentations and LOS. This study has a number of clinical implications and has informed the development of targeted hospital avoidance strategies. Specifically, the finding that females and those diagnosed with personality disorder are more likely to present multiple times has informed the development of an outpatient clinic for people with personality disorder. The finding that over $20 \%$ of $\mathrm{MH}$ presentations were brought in involuntarily by either police or ambulance has informed discussions around the development of a joint response models between police, ambulance and mental health to manage crisis in the community and reduce transportation to the ED where possible. The finding that nearly $15 \%$ of $\mathrm{MH}$ presentations are for comorbid $\mathrm{D} \& \mathrm{~A}$ issues has led to a recommendation that $\mathrm{ED}$ mental health nurses receive D\&A training or that more D\&A clinicians are employed in the ED to work with this population. This study has also called for more expertise in working with children and young people in crisis in the ED.

\section{Conclusion}

This paper examines the profile and journey of patients with mental illness who present to the ED. This study found that females were more likely to present to ED for mental health reasons, and significantly more likely to present multiple times. Presenting issues were associated with age and gender for most diagnostic groups, but not with number of presentations, except for personality disorder. One in five presentations were brought in involuntarily by police or ambulance, of which a third were admitted to a MHIPU. This is higher than the overall cohort, of which just under a quarter were admitted for psychiatric care. Just over half of presentations were referred to a community team for follow up, but only thirty percent were seen by a community team within six months of the ED presentation. This suggests that more needs to be done to improve follow up. There is limited evidence around the patient profile and journey of people in who present to EDs with mental illness, and this study contributes to this literature. 


\section{References}

1. Coates D. An Overview of Service Models for Urgent and Emergency Psychiatric Care. Journal of Psycholsocial Nursing and Mental Health Services. 2018.

2. Morphet J, Munro I, O'Brien A, Gaskin C, Reed F. Managing people with mental health presentations in emergency departments-A service exploration of the issues surrounding responsiveness from a mental health care consumer and carer perspective. . Australasian Emergency Nursing Journal 2012;15:148-55.

3. Sunderji N, Tan de Bebiana J, Stergiopoulos V. Urgent Psychiatric Services: A Scoping Review. Psychiatry 2015;60(9):393-402.

4. Althaus F, Paroz S, Hugli O, Ghali W, Daeppen J, Peytremann-Bridevaux I, et al. Effectiveness of interventions targeting frequent users of emergency departments: A systematic review. Ann Emerg Med. 2011;58(1):41-52.

5. Vandyk A, Harrison M, VanDenKerkhof E, Graham I, Ross-White A. Frequent emergency department use by individuals seeking mental healthcare: a systematic search and review. F Arch Psychiatr Nurs. 2013;27(4):171-8.

6. Australian Institute of Health and Welfare. Mental health services provided in emergency departments 2017 [27/11/2017]. Available from:

https://mhsa.aihw.gov.au/services/emergency-departments/.

7. Hefflefinger L. Transforming psychiatric care delivery in the emergency department: One hospital's journey. Journal of Emergency Nursing. 2014;40(4):365-70.

8. Tankel A, Di Palma M, Kramer K, van der Zwan R. Increasing impact of mental health presentations on New South Wales public hospital departments 1999-2006. Emerg Med Australas. 2011;23:689-96.

9. Shafiei T, Gaynor N, Farrell G. The characteristics, management and outcomes of people identified with mental health issues in an emergency department, Melbourne, Australia. Journal of Psychiatric \& Mental Health Nursing. 2011;18:9-16.

10. Lee S, Thomas P, Doulis C, Bowles D, Henderson K, Keppich-Arnold S, et al. Outcomes achieved by and police and clinician perspectives on a joint police officer and mental health clinician mobile response unit. International Journal of Mental Health Nursing. 2015;24:5385646 .

11. Bost N, Crilly J, Wallen K. The impact of a flow startegy for patients who presented to an Australian emergency department with a mental illness. International Emergency Nursing. 2015;23:265-73.

12. Knott J, Pleban A, Taylor D, Castle D. Management of mental health patients attending Victorian emergency departments. The Royal Australian and New Zealand College of Psychiatrists. 2007;41:759-67.

13. Clarke D, Dusaome D, Hughes L. Emergency department from the mental health client's perspective. International journal of mental health nursing. 2007;16:126-31. 14. Frank R, Fawcet L, Emmerson B. Development of Australia's first psychiatric emergency centre. . Australasian Psychiatry. 2005;13:266 - 72. 
15. Wand T, D'Abrew N, Acret L, White K. Evaluating a new model of nurse-led emergency department mental health care in Australia. International Emergency Nursing. 2016;24:16-21.

16. Paton F, Wright K, Ayre N. Improving outcomes for people in mental health crisis: a rapid synthesis of the evidence for available models of care. Health Technol Assess. 2016;1(161).

17. Janssens A, Hayen S, Walraven V, Leys M, Deboutte D. Emergency psychiatric care for children and adolescents: a literature review. Pediatr Emerg Care. 2013;29(9):1041-50.

18. Lui S, Ali S, Rosychuk R, Newton A. Characteristics of children and youth who visit the emergency department for a behavioural disorder. J Can Acad Child Adolesc Psychiatry. 2014;23(2):111-7.

19. Penfold S, Groll D, Mauer-Vakil D, Pikard J, Yang M, Nadeem Mazhar M. A retrospective analysis of personality disorder presentations in a Canadian universityaffiliated hospital's emergency department. BJPsych Open. 2016;2:394-9.

20. Weiss A, Chang G, Rauch S, Smallwood J, Schechter M, Kosowsky J, et al. Patients and practice related determinants of emergency department length of stay for patients with psychiatric illness. Annals of Emergency Medicine. 2012;60:162-71.

21. Roland M, Abel G. Reducing emergency admissions: are we on the right track? BMJ : British Medical Journal. 2012;345.

22. eHealth NSW. Electronic Medical Record 2017 [Available from: http://www.ehealth.nsw.gov.au/programs/clinical/emr-connect/emr.

23. Hansen D, Kemp M, Mills S, Mercer M, Frosdick P, Lawley M. Developing a national emergency department data reference set based on SNOMED CT. Medical Journal of Australia. 2011;194((4 Suppl): S8.).

24. Bland J, Altman D. Multiple significance tests: the Bonferroni method. 310. $1995 ; 170$ (BMJ).

25. Hosmer D, Lemeshow S. Applied Logistic Regression. 2nd Edition ed. New York, USA. : Wiley and Sons.; 2000.

26. Pregibon D. Logistic regression diagnostics. Annals of Statistics. 1981;9:705-24.

27. NSW Health. Human Research Ethics Committees - Quality Improvement \& Ethical Review: A Practice Guide for NSW. Document number GL2007_020. North Sydney: NSW Health; 2007.

28. Health Records and Information Privacy Act. NSW Legislation No 71: NSW Government; 2002 [Available from:

http://www.legislation.nsw.gov.au/\#/view/act/2002/71/full.

29. National Health and Medical Research Council. Ethical Considerations in Quality Assurance and Evaluation Activities 2014 [Available from:

https://www.nhmrc.gov.au/ files nhmrc/publications/attachments/e111 ethical consider ations in quality assurance 140326.pdf.

30. Australian Bureau of Statistics. 2016 Census QuickStats 2016 [Available from: http://stat.abs.gov.au/itt/r.jsp?databyregion.

31. Lee J, Korczak D. Emergency physician referrals to the pediatric crisis clinic: reasons for referral, diagnosis and disposition. . Can Acad Child Adolesc Psychiatry. 2010;19(4):297302.

32. Indig D, Copeland J, Conigrave KM. Characteristics and comorbidity of drug and alcohol-related emergency department presentations detected by nursing triage text. Addiction (Abingdon, England). 2010;105:897-906. 
33. Butler K, Reeve R, Arora S, Viney R, Goodall S, Van Gool K, et al. The hidden cost of drug and alcohol use in hospital. Drug and alcohol review. 2016;35:359-66.

34. Butler K, Reeve R, Viney R, Burns L. Estimating prevalence of drug and alcohol presentations to hospital emergency departments in NSW, Australia: impact of hospital consultation liaison services. Public Health Research and Practice Perspctives. 2016.

35. Booth B, Walton M, Barry K, Cunningham R, Chermack S, Blow F. Substance Use, Depression, and Mental Health Functioning in Patients Seeking Acute Medical Care in an Inner-City ED. Journal of Behavioral Health Services \& Research. 2011;38(3):358-72.

36. Latt N, Jurd S, Tennant C, Lewis J, Macken L, Joseph A, et al. Alcohol and substance use by patients with psychosis presenting to an emergency department: changing patterns. Australasian Psychiatry. 2011;19(4):354-9.

37. Eaton N, Krueger R, Skodol A, Grant B, Katherine K, Balsis S, et al. An Invariant Dimensional Liability Model of Gender Differences in Mental Disorder Prevalence: Evidence From a National Sample. Journal of Abnormal Psychology. 2011:282-8.

38. Kessler RC, McGonagle KA, Zhao S, Nelson CB, Hughes M, Eshleman S. Lifetime and 12 month prevalence of DSM-III-R psychiatric disorders in the United States: Results from the national comorbidity survey. Archives of General Psychiatry. 1994;51:8-19.

39. Ochoa S, Usall J, Cobo J, Labad X, Kulkarni J. Gender Differences in Schizophrenia and First-Episode Psychosis: A Comprehensive Literature Review. Schizophrenia Research and Treatment. 2012.

40. beyondblue. Men's Help Seeking Behaviour 2012 [Available from:

https://www.beyondblue.org.au/about-us/research-projects/research-projects/men-s-helpseeking-behaviour.

41. Coates D, Saleeba C, Howe D. Mental health attitudes and beliefs in a community sample on the Central Coast in Australia: Barriers to help seeking. Community Mental Health. in press.

42. National Health and Medical Research Council. Clinical Practice Guideline for the Management of Borderline Personality Disorder. Canberra: National Health and Medical Research Council; 2013.

43. Herrington V. Inter-agency Cooperation and Joined-up Working in Police Responses to Persons with a Mental Illness: Lessons from New South Wales. Policing: A Journal of Policy \& Practice. 2012;6(4):388-97.

44. Hollander $Y$, Lee $S$, Tahtalian S. Challenges Relating to the Interface Between Crisis Mental Health Clinicians and Police When Engaging with People with a Mental Illness. Psychiatry, Psychology and Law. 2012;19(3):402-11. .

45. Huppert D, Griffiths M. Police Mental Health Partnership project: Police Ambulance Crisis Emergency Response (PACER) model development. Australasian Psychiatry 2015;23(5):520-23.

46. Kisely S, Campbell LA, Peddle S. A controlled before-and-after evaluation of a mobile crisis partnership between mental health and police services in Nova Scotia. Canadian Journal of Psychiatry. 2010;55:662-8.

47. Bost N, Crilly J, Wallen K. Characteristics and process outcomes of patients presenting to an Australian emergency department for mental health and non-mental health diagnoses. International Emergency Nursing. 2014;22:146-52.

48. Wand T, D'Abrew N, Barnett C, Acret L, White K. Evaluation of a nurse practioner-led extended hours mental health liaison nurse service based in the Emergency Department. Australian Health Review. 2015;39:1-8. 
49. Atzema C, Schiull M, Kurdyak P, Menezes N, Wilton A, Vermeulen M, et al. Wait times in the emergency department for patients with mental illness. CMAJ.

2012;184(18):E969-E79. 